\title{
Soluble receptor for advanced glycation end products in late- onset neonatal infection
}

\author{
Zolakova $\mathrm{B}^{1}$, Zolak $\mathrm{V}^{2}$, Hatok $\mathrm{J}^{3}$, Matasova $\mathrm{K}^{1}$, Nosal $\mathrm{S}^{2}$, Zibolen $\mathrm{M}^{1}$ \\ Department of Neonatology, Jessenius Faculty of Medicine in Martin, Comenius University Bratislava, \\ University Hospital in Martin, Slovakia. bzolakova@gmail.com
}

\section{ABSTRACT}

OBJECTIVES: To assess the plasma levels of SRAGE (soluble receptor for advanced glycation end products) in infected and non-infected preterm neonates and to compare their diagnostic values with standard infection biomarkers.

BACKGROUND: RAGE activates pathways responsible for acute and chronic inflammation. The soluble type of this receptor, SRAGE, which acts as a decoy receptor, has been linked to the severity of sepsis and its outcome. METHODS: Prospective clinical study was carried out from January 2011 to August 2013. There were 33 neonates included according to their infection status and divided into subgroups as follows: infected (I), septic (S), non-infected controls (C).

RESULTS: We found significantly lower values of SRAGE in the subgroup $S(905.54 \pm 220.53 \mathrm{pg} / \mathrm{mL} ; \mathrm{p}<0.028)$, while borderline values were higher in the subgroup I vs C $(2158.33 \pm 197.33 \mathrm{pg} / \mathrm{mL}$ vs $1744.80 \pm 157.74 \mathrm{pg} / \mathrm{mL}$; $p<0.064)$. By analysing the interobserver concordance we detected $70 \%$ agreement as to sRAGE values detected in neonatal late-onset infections and sepsis, while procalcitonin was used as golden standard.

CONCLUSION: Plasma SRAGE values reflect the severity of the inflammatory status in late-onset infection and sepsis in preterm neonates. Our results indicate that SRAGE could be a good potential biomarker of late-onset neonatal infection and sepsis (Tab. 2, Ref. 14). Text in PDF www.elis.sk. KEY WORDS: SRAGE, biomarker, preterm neonate, late-onset infection, late-onset sepsis.

List of abbreviations: $\mathrm{C}$ - controls, CRP - C-reactive protein, ELISA - enzyme-linked immunosorbent assay, HMGB1 - high mobility group box protein 1, I - infected subgroup, JFM CU Jessenius Faculty of Medicine of the Comenius University, Lkc - white blood cells, n - count, NFkB - nuclear factor kappa B, $\mathrm{PCT}$ - procalcitonin, RAGE - receptor for advanced glycation end products, $\mathrm{S}$ - septic subgroup, SD - standard deviation, sRAGE soluble receptor for advanced glycation end products.

${ }^{1}$ Department of Neonatology, Jessenius Faculty of Medicine in Martin, Comenius University Bratislava, University Hospital in Martin, Slovakia, ${ }^{2}$ Department of Paediatric Anaesthesiology and Intensive Medicine, Jessenius Faculty of Medicine in Martin, Comenius University Bratislava, University Hospital in Martin, and ${ }^{3}$ Department of Medical Biochemistry, Jessenius Faculty of Medicine in Martin, Comenius University Bratislava

Address for correspondence: B. Zolakova, MD, Clinic of Neonatology, JFM CU and UH in Martin, Kollarova 2, SK-036 59 Martin, Slovakia. Phone: +421.905 .520307$

Acknowledgement: This work was supported by project "Support of human resources development using the most modern methods and forms of education on JFM CU in Martin" co-financed by EU sources and European Social Fund. We thank all the research staff of Department of Neonatology JFM CU and UH Martin who worked on recruitment and sample collection, as well as staff at the Department of Clinical Biochemistry UHM, and participating families. This work was supported by project "Support of human resources development using the most modern methods and forms of education on JFM CU in Martin" co-financed by EU sources and European Social Fund.

\section{Introduction}

Bacterial infection and sepsis are some of the major causes of neonatal morbidity and mortality especially in preterm infants (1). Prevention and control of the infection are big challenges for neonatal intensive care units. This unmet medical need has inspired a great deal of work to understand the molecular pathogenesis of sepsis and to develop an improved therapeutic intervention. One molecule that has been implicated in the pathogenesis of sepsis is the receptor for advanced glycation end products (RAGE), a member of the immunoglobulin superfamily. Beside advanced glycation end products, this receptor can bind various ligands, newly known as alarmins (HMGB1 - high mobility group box protein, S100 proteins and others) which play important roles in promoting and continuing the host inflammatory response. Therefore RAGE can be counted among the pattern recognition receptors (2).

RAGE-ligand interaction leads to the activation of several transcription factors, including nuclear factor kappa B (NFkB) and mitogen-activated protein kinase, as well as to oxidative stress generation. It results in sustained up-regulation of cytokines, adhesion molecules, and proinflammatory mediators. Thus, the RAGE-ligand interaction leads to a potent immune response (3). In addition to its full-length or membrane-bound form, a number of isoforms of RAGE have been found. Soluble RAGE (sRAGE) is produced by alternative splicing or proteolytic cleavage and its function depends upon the extracellular milieu and severity 
of the inflammatory responses (4). As the sRAGE comprises an extracellular ligand-binding domain by lacking the cytosolic and transmembrane domains, it has the same ligand binding specificity, and therefore competes with cell-bound RAGE, serves as a decoy abrogating cellular activation and ensures a negative feedback in RAGE activity modulation (5). sRAGE plays an important role in inflammation and higher levels of sRAGE are believed to reduce the pathologic effect mediated by RAGE activation $(6,7)$.

Our objective was to determine the sRAGE values in infected preterm infants in dependence of the severity of infection (infection or sepsis) and compare the sRAGE values with the routinely used inflammatory markers (C-reactive protein - CRP, procalcitonin - PCT, total leukocyte count - Lkc) in infected and healthy preterm infants.

\section{Materials and methods}

Patients admitted to the Department of Neonatology, Jessenius Faculty of Medicine, Comenius University (JFM CU) and University hospital in Martin were enrolled between January 2011 and August 2013 with parent's written confirmed consent. JFM CU Ethical Committee approval (EK 650/2010) was taken before the commencement of the study.

\section{Inclusion criteria}

Preterm neonates (born at 24-36 gestational weeks) with clinical signs suggestive for a late-onset infection (after 3 days) were eligible for the study. The entry criteria were physical signs of infection or rapid deterioration of respiratory and cardiovascular functions. Physical symptoms of infection were defined by the presence of at least three of the following: disordered peripheral circulation (peripheral cyanosis, paleness, mottled skin, delayed capillary refill $>3$ seconds), irritability or lethargy, feeding intolerance, abdominal distension, temperature instability, and hepatosplenomegaly. Cardiovascular dysfunction was established by the presence of tachycardia (heart rate $>170$ beats $/ \mathrm{min}$ ) or bradycardia ( $<90$ beats $/ \mathrm{min}$ ) and altered peripheral circulation. Tachypnoea ( $>70$ breaths $/ \mathrm{min}$ ), or episodes of apnoea and increase in the fraction of inspired oxygen were regarded as symptoms of respiratory dysfunction.

\section{Exclusion criteria}

Neonates with congenital anomalies, chromosomal abnormalities, inborn errors of metabolism, confirmed intrauterine viral infection, bronchopulmonary dysplasia, intraventricular haemorrhage, infants who had just undergone surgery and those who had already been receiving parenteral antibiotics at the time of infection evaluation were excluded from the study.

Biologic material was taken in case of any suspicion of infection. Beside the blood count examination, biochemical parameters (CRP, PCT) were investigated, blood was cultured, and a $0.5 \mathrm{~mL}$ blood sample for sRAGE estimation was drawn and consecutively centrifuged. Plasma was stored at $-80^{\circ} \mathrm{C}$ until analysis. In respect to the character of the infection, further indicated biologic material was taken for microbiologic evaluation. Plasma sRAGE concen- trations were measured using an RD191116200R Human sRAGE ELISA (enzyme-linked immunosorbent assay) kits (BioVedor Laboratorní medicína, a.s., Brno, Czech Republic). Intra-assay and inter-assay coefficients of variability were both less than $10 \%$.

There were 33 preterm neonates enrolled, 18 males and 15 females. Mean gestation age was $30.35 \pm 3.2$ weeks, mean birth weight $1,625.88 \pm 556.84 \mathrm{~g}$ and mean length $39.64 \pm 3.95 \mathrm{~cm}$. Enrolled neonates were divided into three subgroups:

- Infants with late-onset infection(I): Neonates with clinical syndrome associated with a high probability of infection, positive finding on examination, imaging or laboratory or bacteriological tests, but negative haemoculture $(\mathrm{n}=10)$.

- Infants with late-onset sepsis (S): Infected infants with cultureproven sepsis $(\mathrm{n}=7)$.

- Control group (C): Neonates without clinical signs of infection and antibiotic treatment $(\mathrm{n}=16)$.

For more details about studied subgroups see Table 1.

\section{Statistical methods}

All data were analyzed with SPSS statistical data software (Chicago, IL, v. 22.0). Continuous variables are expressed as means \pm SD (standard deviation), median, minimum and maximum values using descriptive statistics. All variables were tested on normal distribution prior to analysis. Standard Mann-Whitney nonparametric test and Kruskal-Wallis test, if appropriate, were used with $p$ value of 0.05 considered statistically significant. In interobserver analysis the $\kappa$-coefficient was also calculated in $95 \%$ CI.

Tab. 1. Classification of the studied subgroups.

\begin{tabular}{|c|c|c|c|}
\hline & $\begin{array}{c}\mathrm{I} \\
\mathrm{n}=15\end{array}$ & $\begin{array}{c}\mathrm{S} \\
\mathrm{n}=20\end{array}$ & $\begin{array}{c}C \\
n=14\end{array}$ \\
\hline Males & 5 & 4 & 9 \\
\hline Females & 5 & 3 & 7 \\
\hline \multicolumn{4}{|l|}{ Gestational week } \\
\hline Mean & 29.4 & 31.7 & 32.4 \\
\hline Median & 29.5 & 32.0 & 32.5 \\
\hline SD & 1.03 & 1.04 & 1.5 \\
\hline \multicolumn{4}{|l|}{ Birth weight (g) } \\
\hline Mean & 1390.0 & 1962.9 & 1895.3 \\
\hline Median & 1425.0 & 1900.0 & 1980.2 \\
\hline SD & 137.4 & 213.1 & 210.3 \\
\hline \multicolumn{4}{|l|}{ Trophic } \\
\hline Appropriate & 9 & 6 & 16 \\
\hline Small & 1 & 1 & 0 \\
\hline$\underline{\text { Large }}$ & 0 & 0 & 0 \\
\hline Age at enrolling & (days) & (days) & (days) \\
\hline Mean & 16.6 & 12.7 & 24 \\
\hline Median & 13.6 & 12 & 19 \\
\hline $\mathrm{SD}$ & 5.1 & 2.4 & 2.8 \\
\hline \multicolumn{4}{|l|}{ Mode of delivery } \\
\hline Spontaneous & 2 & 3 & 8 \\
\hline Caesarean & 8 & 4 & 8 \\
\hline Forceps & 0 & 0 & 0 \\
\hline Apgar in 1 th $\operatorname{Min} \leq 7$ & 8 & 4 & 7 \\
\hline$\leq 5$ & 2 & 2 & 2 \\
\hline Apgar in 5 th $\operatorname{Min} \leq 7$ & 5 & 3 & 5 \\
\hline$\leq 5$ & 2 & 1 & 1 \\
\hline
\end{tabular}


Tab. 2. Plasma sRAGE values, standard inflammation markers and microbiology used in the studied subgroups.

\begin{tabular}{|c|c|c|c|c|}
\hline & \multicolumn{3}{|c|}{ Subgroup } \\
\hline & & I & $S$ & $\mathrm{C}$ \\
\hline \multirow[t]{6}{*}{ Age (days) } & Mean & 16.6 & 12.71 & 23.0 \\
\hline & SD & 5.07 & 2.41 & 2.79 \\
\hline & Median & 13.00 & 12.00 & 19.00 \\
\hline & Minimum & 1.00 & 32.00 & 11.00 \\
\hline & Maximum & 55.00 & 26.00 & 49.00 \\
\hline & Range & 59.59 & 19.00 & 38.00 \\
\hline \multirow{6}{*}{$\begin{array}{l}\text { sRAGE } \\
(\mathrm{pg} / \mathrm{mL})\end{array}$} & Mean & $2,158.33$ & 905.54 & $1,744.80$ \\
\hline & SD & 197.63 & 220.53 & 157.74 \\
\hline & Median & $2,277.08$ & 651.40 & $1,716.48$ \\
\hline & Minimum & 1378.32 & 199.53 & 756.80 \\
\hline & Maximum & $3,014.36$ & $1,800.12$ & $3,491.61$ \\
\hline & Range & $1,636.04$ & $1,600.59$ & $2,734.81$ \\
\hline \multirow{6}{*}{$\operatorname{Lkc}\left(\mathrm{x} 10^{9}\right)$} & Mean & 12.70 & 13.32 & 11.04 \\
\hline & SD & 1.30 & 2.81 & .85 \\
\hline & Median & 12.45 & 12.22 & 10.60 \\
\hline & Minimum & 7.78 & 6.20 & 3.10 \\
\hline & Maximum & 19.40 & 29.00 & 17.40 \\
\hline & Range & 11.62 & 22.80 & 14.30 \\
\hline \multirow[t]{6}{*}{$\overline{\mathrm{CRP}}(\mathrm{mg} / \mathrm{L})$} & Mean & 22.54 & 74.63 & .50 \\
\hline & SD & 12.62 & 25.82 & .25 \\
\hline & Median & 6.30 & 33.50 & .10 \\
\hline & Minimum & .98 & 14.10 & .10 \\
\hline & Maximum & 132.00 & 169.00 & 3.90 \\
\hline & Range & 131.02 & 154.90 & 3.80 \\
\hline \multirow[t]{6}{*}{ PCT (ng/mL) } & Mean & 6.27 & 12.17 & .13 \\
\hline & SD & 5.75 & 7.98 & .01 \\
\hline & Median & .48 & 4.50 & .12 \\
\hline & Minimum & .00 & .00 & .08 \\
\hline & Maximum & 58.00 & 59.18 & .20 \\
\hline & Range & 58.00 & 59.18 & .12 \\
\hline \multirow[t]{2}{*}{ Microb. } & Negative $\mathrm{N}$ & 2 & 0 & 16 \\
\hline & Positive $\mathrm{N}$ & 8 & 7 & 0 \\
\hline
\end{tabular}

SD - standard deviation, I - infected subgroup, $\mathrm{S}$ - septic subgroup, $\mathrm{C}$ - controls, sRAGE - soluble receptor for advanced glycation end products, Lkc- white blood cells, CRP - C-reactive protein, PCT - procalcitonin, $\mathrm{N}$ - count

\section{Results}

In all our studied subgroups (I, S, C), standard inflammation markers were examined as follows: CRP, PCT, Lkc, microbiology culture, and sRAGE. For more details see Table 2.

The highest mean plasma sRAGE values were found in the subgroup I, the lowest mean values were in the subgroup S. We have demonstrated a statistically significant difference in sRAGE distribution between studied subgroups $(\mathrm{p}<0.001)$. Detailed pairwise analysis showed differences in sRAGE concentrations between studied subgroups as follows: sRAGE values in the subgroup I were borderline higher to those in subgroup C, $(2158.33 \pm 197.33 \mathrm{pg} / \mathrm{mL}$ vs $1744.80 \pm 157.74 \mathrm{pg} / \mathrm{mL} ; \mathrm{p}<0.064)$; sRAGE values in the subgroup S were significantly lower compared to subgroup C $(905.54 \pm 220.53$ $\mathrm{pg} / \mathrm{mL}$ vs $1744.80 \pm 157.74 \mathrm{pg} / \mathrm{mL} ; \mathrm{p}<0.028)$. There was a significant difference in sRAGE values between the subgroups I and $\mathrm{S}(2158.33 \pm 197.33 \mathrm{pg} / \mathrm{mL}$ vs $905.54 \pm 220.53 \mathrm{pg} / \mathrm{mL} ; \mathrm{p}=0.004)$.

We have demonstrated a statistically significant difference in PCT and CRP distribution between studied subgroups for both ( $\mathrm{p}$ $<0.001)$. A detailed pairwise comparison showed results as fol- lows: CRP values were significantly higher in subgroup I compared to subgroup C $(22.54 \pm 12.62 \mathrm{mg} / \mathrm{mL}$ vs $0.50 \pm 0.25 \mathrm{mg} / \mathrm{mL} ; \mathrm{p}<$ $0.001)$ and in subgroup $\mathrm{S}$ compared to subgroup $\mathrm{C}(74.63 \pm 25.82$ $\mathrm{mg} / \mathrm{mL}$ vs $0.50 \pm 0.25 \mathrm{mg} / \mathrm{mL} ; \mathrm{p}<0.001)$. There was no difference in CRP values between subgroups I and S $(22.54 \pm 12.62 \mathrm{mg} / \mathrm{mL}$ vs $74.63 \pm 25.82 \mathrm{mg} / \mathrm{mL} ; \mathrm{p}=0.139$ ). For PCT we obtained similar results: in the subgroup I, the PCT values were significantly higher compared to those in subgroup $\mathrm{C}(6.27 \pm 5.75 \mathrm{ng} / \mathrm{mL}$ vs $0.13 \pm 0.01$ $\mathrm{ng} / \mathrm{mL} ; \mathrm{p}<0.015)$ and in subgroup $\mathrm{S}$ they were significantly higher compared to subgroup C $(12.17 \pm 7.98 \mathrm{ng} / \mathrm{mL}$ vs $0.13 \pm 0.01 \mathrm{ng} / \mathrm{mL}$, $\mathrm{p}<0.001)$. We did not find any statistical difference in PCT distribution between I and S subgroups $(6.27 \pm 5.75 \mathrm{ng} / \mathrm{mL}$ vs $12.17 \pm 7.98$ $\mathrm{ng} / \mathrm{mL} ; \mathrm{p}=0.105)$. We did not find a significant difference in Lkc distribution between studied subgroups $(p=0.111)$.

For expression of the power of each inflammatory biochemical marker we used interobserver analysis test with estimation of coefficient- $\kappa$. РCT was used as golden standard, because of its highest sensitivity for the infected infants' subgroup. We detected concordance with sRAGE in infected patients as follows: $70 \%$ for subgroup $\mathrm{I}(\kappa=0.4)$, and $71.4 \%$ for $\mathrm{S}(\kappa=0.3)$. Interestingly, by means of CRP assessment we diagnosed $60 \%$ of late-onset infections $(\kappa=0.167)$ and $93 \%$ of late-onset sepsis $(\kappa=0.63)$.

\section{Discussion}

In our study we estimated plasma sRAGE values and routinely used inflammatory markers in healthy and infected preterm infants before starting the antibiotic treatment. The infected infants without bacteraemia had higher sRAGE values compared to controls (borderline statistical difference). It is possible that the neonatal innate immune system uses the soluble RAGE for the circulating membrane RAGE receptor ligands neutralisation, and so provides a competitive inhibition of the proinflammatory effects related to the membranous RAGE. Therefore during the inflammatory response in infected infants, the levels of sRAGE increase. We obtained analogous results by assessment of sRAGE values in cases of early-onset infection in term neonates (unpublished results).

In contrast, sRAGE values were significantly decreased in cases of bacteriologically proven sepsis. We detected significantly lower plasma sRAGE levels in the subgroup of preterm infants with late-onset sepsis compared to controls. However, by massive cytokinaemia during bacteraemia, the immature organism cannot produce a satisfying amount of neutralising forms, and that is why a decrease in SRAGE takes place and sepsis develops.

Buhimschi and colleagues found a significant inverse relationship between the foetal systemic levels of sRAGE and severity of inflammation in the maternal and foetal compartments (8). Likewise, infants with very low birth weight with funisitis had significantly lower sRAGE values in cord blood compared to the healthy infants (9). Bastek and colleagues found significantly lower cord blood sRAGE in infants with early-onset sepsis (10). The levels of sRAGE were significantly decreased in foetuses and neonates with a mounting inflammatory reaction. Thereby, it is possible that sRAGE consumption allows an excessive activation of multiple signal transduction pathways leading to tissue damage 
and multiple organ failure in infants with a robust inflammatory response to infection.

RAGE ligands accumulation during sepsis leads to sRAGE consumption and consecutive decrease in neonates. Low plasma sRAGE values by inflammatory response reflect a less regulated immune response and account for higher risk of the negative outcome. As sRAGE acts as a "decoy" for RAGE, it may be assumed, that a decrease in circulating sRAGE takes place in the milieu of a robust inflammatory response. It may be a result of inhibition or dysfunction mechanisms responsible for this neutralising ligand synthesis during massive inflammatory response accompanying the system inflammatory response with bacteraemia. Low sRAGE levels may reflect successful detoxification of ligands generated during higher metabolic requirement and oxidative stress coupled with immaturity and infection with bacteraemia. We did not asses the infants' outcome in relation to sRAGE decrease during sepsis.

Rogers and colleagues elicit, parallel with us in cases of the late-onset sepsis, a negative correlation between sRAGE values and early-onset sepsis development in extremely preterm infants. In their study, contrary to ours, the blood samples were taken routinely on the 5th or 7th day, but not in the relation to the infectious disease development. Plasma sRAGE values in the studied group did not correlate with such complications as necrotising enterocolitis, intraventricular haemorrhage, periventricular leukomalatia or bronchopulmonal dysplasia (11). There is a lack of studies exploring the relation of RAGE/sRAGE to the later complications of the preterm infants. We excluded infants with the mentioned complications of the preterm control subgroup for their possible impact on sRAGE values. Constantine and his colleagues determined a negative correlation between cord blood SRAGE and preterm infants' mortality (12). In our group, three neonates died; hence it was not possible to evaluate the relation of sRAGE to mortality.

The soluble sRAGE may be comparable with routinely used inflammatory markers. In agreement with our results, some studies showed that PCT provided better diagnostic accuracy than CRP $(13,14)$. By sRAGE we were able to identify $70 \%$ of late-onset neonatal infections and $71.4 \%$ of late-onset sepsis that were diagnosed by golden standard, i.e. by PCT. These results suggest a good diagnostic value of sRAGE in comparison with PCT in the diagnosis of late-onset infection in preterm neonates.

In our study group, routinely used inflammatory markers, CRP and PCT, distinguish well between healthy and ill (infected) neonates. We found no significant difference between infected infants with negative haemoculture and those suffering from sepsis. By comparison of sRAGE values between the subgroups we found that SRAGE can sensitively distinguish between infected and septic infants.

Our results indicate that sRAGE could be a promising biomarker of late-onset neonatal infection and sepsis. For the possible future use of sRAGE in the diagnosis of infection it is necessary to understand better the role of the axis ligand-RAGE-sRAGE in the pathways of neonatal infection. The investigation of plasma or serum biomarkers that could be rapidly detected and might provide risk stratification for preterm neonates could reduce morbidity and provide better outcomes for these most vulnerable patients.

\section{Learning points}

- RAGE activation plays an important role in inflammatory response activation and its perpetuation.

- sRAGE is an endogenous RAGE antagonist and acts as a decoy receptor for RAGE ligands.

- sRAGE values in preterm infected neonates reflect the immune system activity.

\section{References}

1. Shah BA, Padbury JF. Neonatal sepsis. An old problem with new insights. Virulence 2014; 5 (1): $170-178$.

2. Reynolds PR, Smidt RE, Kasteler SD et al. Receptors for advanced glycation end-products targeting protect against hyperoxia-induced lung injury in mice. Am J Respir Cell Mol Biol 2010; 42 (5): 545-551.

3. van Zoelen MAD, Schouten M, de Vos AF et al. The receptor for advanced glycation end products impairs host defense in pneumococcal pneumonia. $\mathrm{J}$ Immunol 2009; 182 (7): 4349-4356.

4. Romero R, Chaiworapongsa T, Savasan ZA et al. Clinical chorioamnionitis is characterized by changes in the expression of the alarmin HMGB1 and one of its receptors, sRAGE. J Matern Fetal Neonat Med 2012; 25 (6): $558-567$.

5. Chuah YK, Basir R, Talib H, Tie TH, Nordin N. Receptor for advanced glycation end products and its involvement in inflammatory diseases. Int $\mathrm{J}$ Inflam 2013; 2013: 403-460.

6. Bopp C, Hofer S, Weitz J et al. sRAGE is elevated in septic patients and associated with patients outcome. J Surg Res 2008; 147 (1): 79-83.

7. Santilli F, Vazzana N, Bucciarelli LG, Davi G. Soluble forms of RAGE in human diseases: clinical and therapeutical implications. Curr Med Chem 2009; 16 (8): 940-952.

8. Buhimschi CS, Baumbuschi MA, Dulay AT et al. Characterization of RAGE, HMGB1, and S100beta in inflammation-induced preterm birth and fetal tissue injury. Am J Pathol 2009; 175 (3): 958-975.

9. Thomas W, Seidenspinner S, Kawczyńska-Leda N, Wirbelauer J, Szymankiewicz M, Speer CP. Soluble receptor for advanced glycation end products (sRAGE) in tracheobronchial aspirate fluid and cord blood of very low birth weight infants with chorioamnionitis and funisitis. Early Hum Dev 2010; 86 (9): 593-598.

10. Bastek JA, Brown AG, Foreman MN, McShea MA, Anglim LM, Adamczak JE, Elovitz MA. The soluble receptor for advanced glycation end products can prospectively identify patients at greatest risk for preterm birth. J Matern Fetal Neonatal Med 2012; 25 (9): 1762-1768.

11. Rogers LK, Graf AE, Bhatia A, Leonhart KL, Oza-Frank R. Associations betweenm maternal and infant morbidities and sRAGE within the first week of life in extremely preterm infants. PLoS ONE 8 (12): e82537.

12. Costantine MM, Weiner SJ, Rouse DJ et al. Umbilical cord blood biomarkers of neurologic injury and the risk of cerebral palsy or infant death. Int J Dev Neurosci 2011; 29 (8): 917-922.

13. Ali AM, Moaz MA, Ghoniem E. Reliability of serum procalcitonin concentrations for the diagnosis of sepsis in neonates. Egypt J Immunol 2008; 15 (1): 75-84.

14. Kordek A, Loniewska B, Podraza W, Nikodemski T, Rudnicki J. Usefulness of estimation of blood procalcitonin concentration versus C-reactive protein concentration and white blood cell count for therapeutic monitoring of sepsis in neonates. Postepy Hig Med Dosw 2014; 21 (68): 1516-1523.

Received April 9, 2015. Accepted June 26, 2015. 